\title{
Intraoperative Images of the Totally Extraperitoneal Repair of Concurrent Spigelian and Groin Hernias
}

\author{
David B. Staab, MD, John D. Welander, MD \\ Department of Surgery, Veterans Affairs Central lowa Health Care System, Des Moines, IA, USA (Dr. Staab). \\ Surgery Residency Program of the lowa Methodist Medical Center, Des Moines, IA, USA (Dr. Welander).
}

\begin{abstract}
Minimally invasive surgical technology is changing the management of spigelian hernias. In addition to open repairs, laparoscopic transabdominal repairs as well as laparoscopic totally extraperitoneal repairs are well documented. However, because spigelian hernias are uncommon, few surgeons have a large volume of experience with spigelian hernia repairs. This is particularly true of the repair of spigelian hernias with concurrent inguinal and femoral hernias. We provide intraoperative images of bilateral spigelian and inguinal and femoral hernias repaired via a laparoscopic totally extraperitoneal approach. This patient was successfully treated with a single piece of mesh on each side. The single mesh piece on the right repaired the right spigelian hernia and a femoral hernia. The single mesh piece on the left repaired the spigelian hernia and left direct and indirect inguinal hernias. Preoperative computed tomography scans illustrate the close proximity of the spigelian defects and the inguinal defects that allow the use of a single $10 \times 15$-cm mesh piece for repair of each side. Our goal is to provide intraoperative images to allow a surgeon who is experienced with totally extraperitoneal repairs to visualize this operation and apply this approach to other patients with concurrent spigelian and inguinal or femoral hernias.
\end{abstract}

Key Words: Spigelian hernia, Laparoscopic, Minimally invasive surgery, Totally extraperitoneal repair, Inguinal hernia, Femoral hernia, Mesh repair.

Citation Staab DB, Welander JD. Intraoperative images of the totally extraperitoneal repair of concurrent spigelian and groin hernias. CRSLS e2014.00119. DOI: 10.4293/JSLS.2014.00119

Copyright $\odot 2014$ SLS This is an open-access article distributed under the terms of the Creative Commons Attribution-Noncommercial-ShareAlike 3.0 Unported license, which permits unrestricted noncommercial use, distribution, and reproduction in any medium, provided the original author and source are credited.

Address correspondence to: David Staab, Veterans Affairs Central Iowa Health Care System, 3600 30th Street, Des Moines, IA 50310, USA. Telephone: (515) 699-5826, Fax: (515) 699-5905, E-mail: David.Staab@VA.gov

\section{INTRODUCTION}

Spigelian hernia repair has changed from being an exclusively open procedure to include the laparoscopic transabdominal preperitoneal ${ }^{1}$ and laparoscopic totally extraperitoneal (TEP) approaches. When spigelian hernias and inguinal hernias occur concurrently, published reports of repairs include open preperitoneal mesh repairs of the spigelian hernia and open tension-free repair of the inguinal hernia. ${ }^{2,3}$ This article will focus on laparoscopic repairs done extraperitoneally: the TEP approach. Publications on the TEP approach include a prospective randomized trial that determined that laparoscopic TEP approach in elective repairs is effective, safe, and less painful. ${ }^{4}$ We found a single publication recommending laparoscopic extraperitoneal repair of concurrent spigelian and inguinal hernias with a single piece of mesh. ${ }^{5}$ Despite quality photographs and line drawings in these publications, we found this repair difficult to visualize, particularly when one examines the patient's anterior abdominal wall during an office visit. It would seem that the spigelian defect and direct, indirect, and femoral hernia defects are too far apart for repair with the standard-sized mesh piece used in TEP repairs of inguinal or femoral hernias. During a recent operation, we obtained intraoperative images that were helpful in visualizing the proximity of spigelian and inguinal/femoral hernias. Our purpose is to present these images in a way that will aid a surgeon who is experienced with the TEP approach to plan for the operation in a patient with concurrent spigelian and inguinal or femoral hernias.

An elderly male patient with bilateral spigelian hernias and concurrent inguinal and femoral hernias presented at our clinic for repair. The patient is a lifelong fitness pro- 


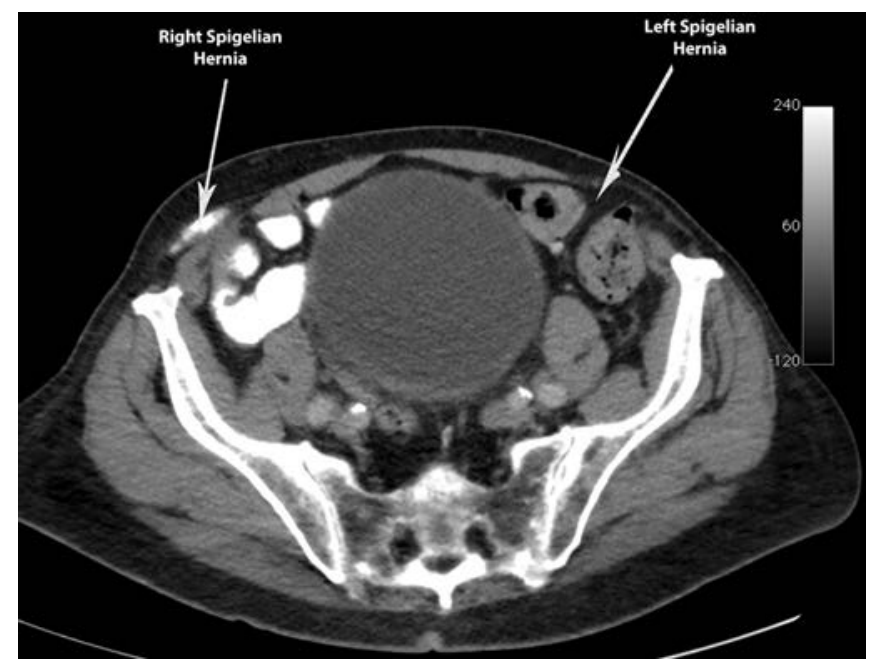

Figure 1. This CT scan image in our patient demonstrates right and left spigelian hernias. The right side defect contains bowel with intraluminal contrast. The left side spigelian defect contains extraperitoneal fat and bowel.

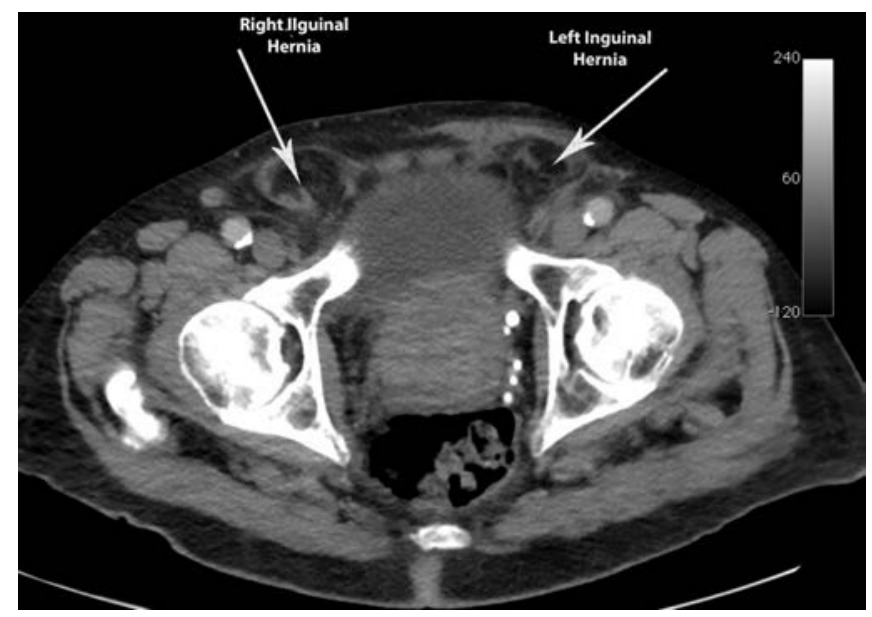

Figure 2. A CT scan image of our patient's right groin hernia, intraoperatively found to be a femoral hernia, and left inguinal hernias.

ponent: actively stair climbing, using a rowing machine, and doing sit-ups on an inclined plane. The patient had noticed his hernias in the previous year and complained of constipation and 2 episodes of vomiting that ceased when the hernias were reduced. He associated his symptoms with the bulges at the spigelian hernia sites. His inguinal and femoral hernias were causing him minimal discomfort but were getting larger and were worrisome to him.

Preoperative computed tomography (CT) scan images were obtained and revealed bilateral spigelian and right and left inguinal hernia defect. The spigelian defects
A

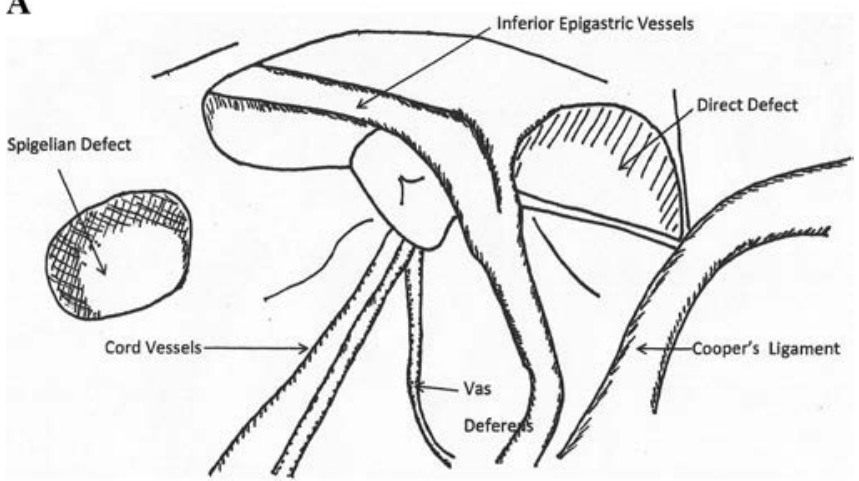

B

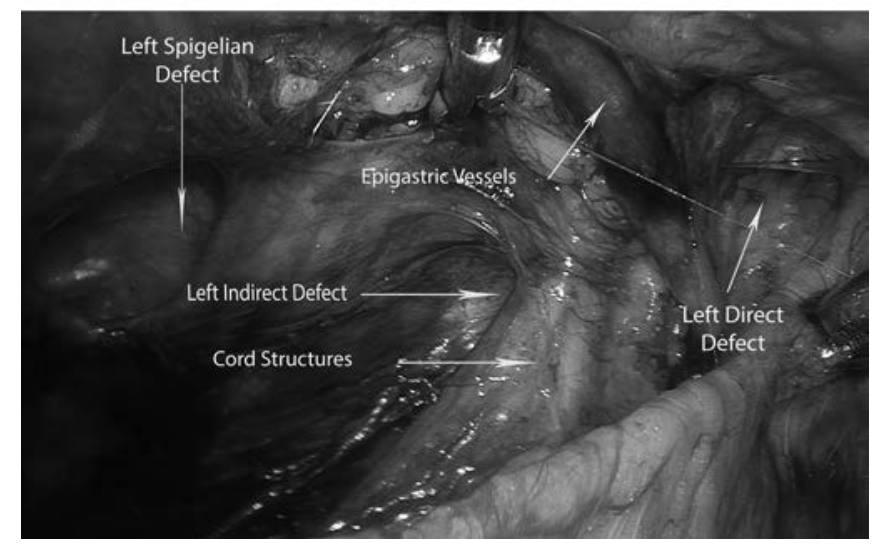

Figure 3. A, Intraoperative photo of the left spigelian hernia and the left indirect and direct hernia defects. Note the proximity of the 3 defects. B, Line drawing with expanded view for labeling purposes. The left Cooper ligament, inferior epigastric vessels, cord vessels, vas deferens, the spigelian defect, and the direct inguinal defect are demonstrated.

measured 3.5 to $4 \mathrm{~cm}$ on the right and $3 \mathrm{~cm}$ on the left. They contained bowel or intra-abdominal fat, see Figure 1. The inguinal defects seen on CT scan are shown in Figure 2. The right side defect was found to be a femoral hernia during the operation. Our radiologist looked at the CT scan axial, sagittal, and coronal views and measured the distance from the groin hernia to the closest edge of the spigelian defect. The estimated distance on the CT scan between the right femoral and right spigelian defects was 5 to $7 \mathrm{~cm}$ and between the left direct inguinal hernia and the left spigelian hernia was $4 \mathrm{~cm}$.

We were aware of published accounts of the TEP repair of concomitant spigelian and inguinal/femoral hernias repair with a single piece of mesh. After discussion, the patient was agreeable to elective TEP repair of his hernias with the possibility of open repair if his hernias were not amenable to TEP repair. 


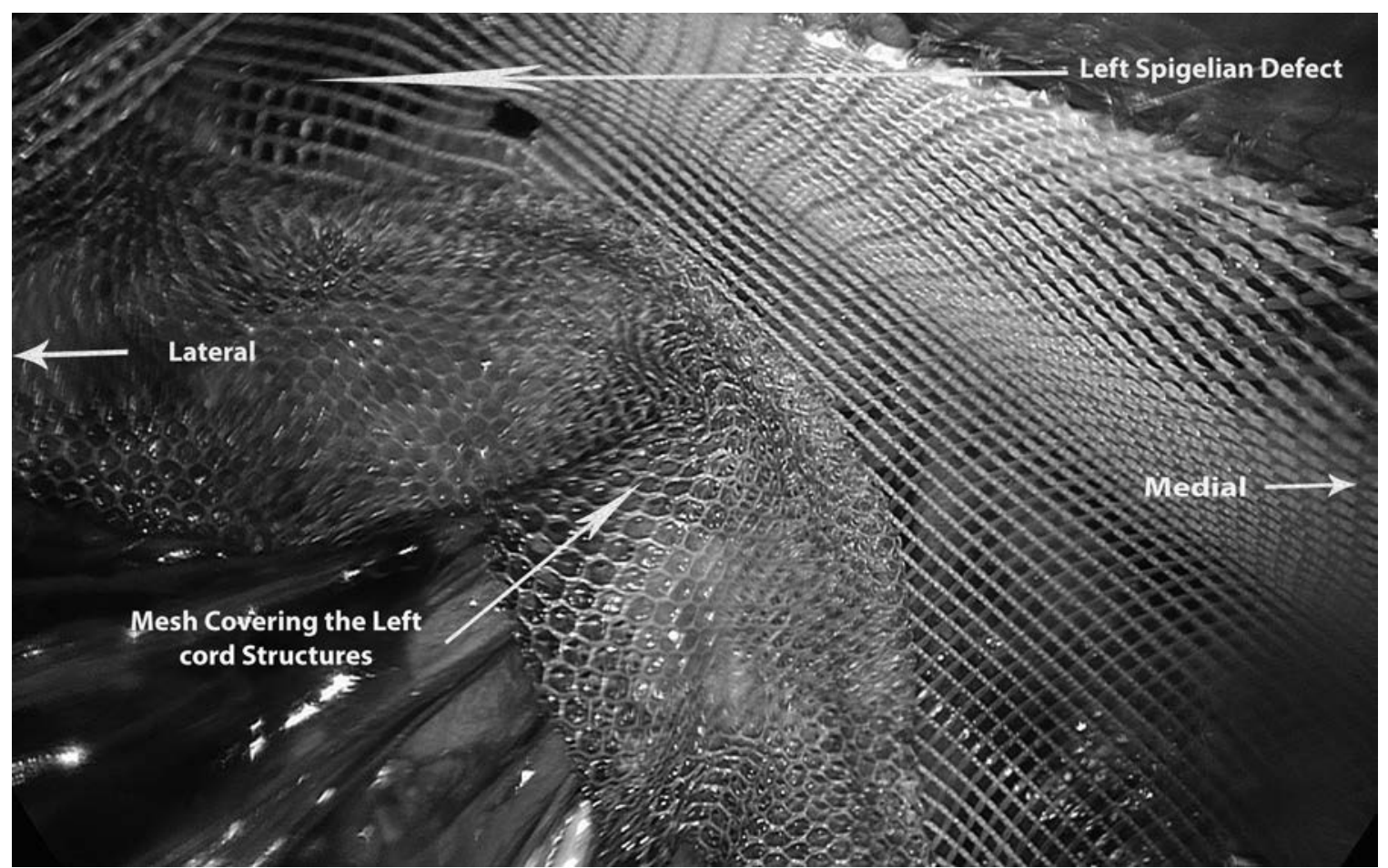

Figure 4. Intraoperative photo of the mesh in place on the left with coverage of the spigelian hernia defect, the indirect defect, the cord structures, and the direct defect seen in Figure 3A. Note coverage of the spigelian and inguinal defects and adequate mesh overlap.

\section{METHODS}

We performed the operation using the standard patient positioning and draping for the extraperitoneal approach. We gained access by making an off-midline incision in the anterior rectus sheath, swept the rectus muscle laterally, and then developed a working space with a balloon dissection device. We used 25 -mm midline ports in the midline for working ports. Laparoscopic exploration and dissection revealed that we were able to visualize and reduce the inguinal defects.

We began the dissection at the spigelian defects. Our technique for reduction of the spigelian hernia was gentle traction circumferentially on the hernia sac near the edge of the fascial defect. We used a blunt dissector for the dissection. We similarly removed the peritoneum from the left side direct and indirect inguinal hernias. The patient had a left direct and indirect inguinal hernia. On the right side, he had a femoral hernia in addition to the spigelian hernia. The left side intraoperative images are shown in Figure 3A and demonstrate the spigelian, direct, and indirect defects. The photographs demonstrate the close proximity of the spigelian fascial defect to the inguinal defects. Figure $3 \mathrm{~B}$ is an expanded line drawing of the left side anatomy. The left side spigelian and inguinal defects were adequately covered with a single $10 \times 15-\mathrm{cm}$ polypropylene mesh, see Figure 4. Mesh fixation was secured with an absorbable tacking device superiorly and inferiorly around the spigelian defects, as well as along the Cooper ligament and near the midline. We elected to place tacks, rather than to use fibrin glue, due to the multiple hernias and the size of the inguinal defects. The repair of the right side was similar to that of the left: a single piece of mesh covered the right femoral defect and the right spigelian hernia defect. We estimated that the repair was completed with 3 to $4 \mathrm{~cm}$ of mesh overlap on the spigelian defects.

The patient then was admitted postoperatively, started on liquids, and sent home the next day using oral acetaminophen for pain control. He was seen in the clinic several weeks postoperatively and was found to have a seroma in his right inguinal hernia site and his left spigelian hernia site. These were not drained and when the patient returned in 3 months, the seromas had resolved, and he had returned to his previous exercise routine. 


\section{DISCUSSION}

Description of TEP repair of concurrent spigelian and inguinal/femoral hernia defects in the literature is limited to several case reports. There is not an extensive collection of data due to the relative rarity of spigelian hernias occurring concurrently with inguinal and femoral hernias. We were pleased with not only the visualization of the anatomy afforded by the extraperitoneal approach, but also with the ease of repair, which does not deviate from our routine TEP repair of inguinal and femoral hernias. We hope the clarity of the images will make the anatomy understandable to the point that surgeons who are experienced with the TEP approach will be able to treat other patients with spigelian hernias using this technique. The patient was operated on 18 months prior to the writing of this article, and he has been satisfied with the repair, the minimal postoperative discomfort, and his ability to resume his workout routine, which is a significant factor in his quality of life.

\section{CONCLUSIONS}

We recommend careful study of pelvic anatomy as seen with the extraperitoneal approach to assist the surgeon in successful repair of spigelian hernias concurrent with inguinal or femoral hernias.

\section{References:}

1. Baucom C, Nguyen QD, Hidalgo M, Slakey D. Minimally invasive spigelian hernia repair. JSLS. 2009;13(2):263-268.

2. Zuvela M, Milicevic M, Galun D, Djuric-Stefanovic A, Bulajic P, Palibrk I. Spigelian hernia repair as a day-case procedure. Hernia. 2013;17(4):483-486.

3. Perrakis, A, Velimezis G, Kapogiannatos G, Koronakis D, Perrakis E. Spigel hernia: a single center experience in a rare hernia entity. Hernia. 2012;16(4):439-444.

4. Moreno-Egea A, Carrasco L, Girela E, Martin JG, Aguayo, JL, Canteras, M. Open vs laparoscopic repair of spigelian hernia: a prospective randomized trial. Arch Surg. 2002;137(11):12661268.

5. Kosal N, Altinli E, Celik A, Oner I. Extraperitoneal laparoscopic approach to spigelian hernia combined with groin hernias. Surg Laparosc Endosc Percutan Tech 2004;14(4):204-206. 